\title{
Resposta funcional e capacidade predatória da fase larval de Ceraeochrysa caligata alimentada com Brevicoryne Brassicae
}

\section{Functional response and predatory ability of the larval stage of Ceraeochrysa caligata fed Brevicoryne brassicae}

\author{
Joel Corrêa de Souza ${ }^{\text {; }}$ "Jessivaldo Rodrigues Galvão ${ }^{2}$; Wilson José de Melo e Silva Maia3; Pedro Paulo da Costa Alves \\ Filho $^{4}$; Antonio Vinicius Corrês Barbosa ${ }^{5}$
}

\begin{abstract}
RESUMO - O pulgão, Brevicoryne brassicae (L.), é uma das principais pragas da cultura da couve. A necessidade de controle de insetos-pragas de forma racional e sustentável tem gerado a busca de medidas efetivas de controle. Objetivou-se estudar a capacidade predatória e a resposta funcional da fase jovem de Ceraeochrysa caligata (Banks) alimentada com o pulgão Brevicoryne Brassicae (L.), usando ninfas de segundo e terceiro instares. Foram utilizadas cinco densidades de presas, com cinco repetições com dois indivíduos por repetição. O Consumo aumentou proporcionalmente em função do estádio de desenvolvimento da larva, sendo significativamente maior no terceiro instar, representando acima de $75 \%$ do total. Os consumos médios diário e totais foram de 4,2 e 25,6; 10,2 e 70,2; 38,6 e 549,0 pulgões, para os três instares, respectivamente. Observou para a fase larval 23,4 e 644,8 pulgões. Constatou se duração média de 5,8; 6,7; 13,5 e de 26,0 dias para o primeiro, segundo e terceiro instares e fase larval, respectivamente. Observou se para os três instares e fase larval, um maior consumo em função do aumento da densidade de presas. A duração obtida para o primeiro e terceiro instares e fase larval, um maior consumo em função do aumento da disponibilidade de presas.
\end{abstract}

Palavras-chave: Insecta. Predação. Resposta funcional. Pulgão da couve.

\begin{abstract}
The aphid, Brevicoryne brassicae (L.), is a major pest of cabbage culture. The need for insect pest control in a rational and sustainable manner has generated the search for effective control measures. The objective was to study the predatory capacity and the functional response of the young phase Ceraeochrysa caligata (Banks) fed with the aphid Brevicoryne brassicae (L.), using nymphs of second and third instars. Five prey densities were used, with five replicates with two individuals per replicate. Consumption increased in proportion to the larval stage of development and was significantly higher in the third instar, representing over $75 \%$ of the total. The daily and total average intake were 4.2 and $25.6 ; 10.2$ and 70.2; Aphids 549.0 and 38.6 for the three instars, respectively. Observed for the larval stage 23.4 and 644.8 aphids. Found a mean duration of $5.8 ; 6.7 ; 13.5$ and 26.0 days for the first, second and third instar and larval stage, respectively. Observed to the three instars and larval stage, a higher consumption due to the increase in prey density. The duration obtained for the first and third instar larval stage and a higher consumption due to the increased availability of prey.
\end{abstract}

Keywords: Insecta. Predation. Functional response. Cabbage aphid.

\footnotetext{
*Autor para correspondência

Recebido para publicação em 03/03/2014; aprovado em 20/07/2015

${ }^{1}$ Joel Corrêa de Souza; Doutor em Ciências Agrárias, Universidade Federal Rural da Amazônia, E-mail: joel.correa@ufra.edu.br; Belém-Pará.

${ }^{2}$ Jessivaldo Rodrigues Galvão; Doutor em Ciências Agrárias, Universidade Federal Rural da Amazônia, E-mail: Jessivaldo.galvao@ufra.edu.br

${ }^{3}$ Wilson José de Melo e Silva Maia; Doutor em Agronomia; Universidade Federal Rural da Amazônia; E-mail: Wilson.maia@ ufra.edu.br

${ }^{4}$ Pedro Paulo da Costa Alves Filho; Graduando em Agronomia; Universidade Federal Rural da Amazônia; E-mail:pp.alvesfilho@ yahoo.com.br

${ }^{5}$ Antônio Vinicius Corrêa Barbosa; Doutor em Geofísica; Universidade Federal Rural da Amazônia; E-mail:vinicius.barbosa@ufra.edu.br
} 


\section{INTRODUÇÃO}

A couve-de-folhas (Brassica oleracea L. var. acephala Brassicaceae), vem expandindo sua área plantada, provavelmente, devido as novas maneiras de utilização na culinária e seu grande valor nutricional (CATI, 2009). A incidência de inúmeras pragas sobre essa cultura induz ao uso intensivo de inseticidas, fato este que preocupa cada vez mais o consumidor, uma vez que esse produto tem grande consumo na forma 'in natura'. Entre os insetos danosos destaca-se o pulgão Brevicoryne brassicae. Face ao exposto, evidencia-se a necessidade de pesquisas de outras medidas de controle. (STEIN \& TEIXEIRA, 2010).

A redução populacional destas pragas é baseada principalmente no método de controle químico com inseticidas de elevada toxicidade (PAZINI \& GALLI, 2011). A utilização inadequada destes inseticidas pode acelerar o processo de desenvolvimento de resistência das pragas, além de ocasionar problemas para o agroecossistema, relacionado ao desequilíbrio e à contaminação ambiental, e à presença residual destas moléculas nos alimentos (LIMA \& GRAVINA, 2009; MOURA et al., 2009; PAZINI \& GALLI, 2011). Com o propósito de minimizar esta problemática, as pesquisas têm se esforçado em busca de métodos de controle ecologicamente eficazes para o manejo populacional destes insetos, o que tem culminado principalmente com o uso de agentes de controle biológico (SOUZA-FILHO et al., 2007; MOURA \& MOURA, 2011). Para o uso do controle biológico é importante o conhecimento das populações dos principais inimigos naturais presentes naturalmente no agroecossistema, visando estabelecer estratégias individuais ou em conjunto com outras táticas de manejo, visando à conservação e multiplicação destes organismos (MOURA \& MOURA, 2011; PAZINI; GALLI, 2011).

A família Chrysopidae se destaca pela riqueza de inimigos naturais de ácaros e insetos-pragas em muitas culturas de interesse econômico, controlando assim a densidade populacional destes organismos. Possui grande capacidade de busca, voracidade das larvas, alto potencial reprodutivo, resistência a certos inseticidas e facilidade de criação em laboratório, o que favorece o uso desses insetos em programas de controle biológico (MAIA et al., 2004).

As larvas de crisopídeos passam por três instares, ocorrendo à última ecdise dentro do casulo. A duração de cada instar e da fase larval é influenciada pela disponibilidade e qualidade do alimento, umidade relativa do ar e pela temperatura (MAIA, 2003). O primeiro instar varia de 2 a 7 dias, o segundo varia de 2 a 5 dias e o terceiro pode se extender em até 10 dias (Fonseca et al., 2000; BOREGAS, 2000). Em função do número de presas consumidas e aquelas disponíveis se pode estabelecer três tipos de resposta funcional Tipo I, Tipo II e Tipo III (MAIA, 2003).

A resposta funcional de um predador descreve a relação entre o número de presas capturadas e o número de indivíduos disponíveis, determinando a quantidade de presas consumidas à medida que a densidade inicial aumenta (SOLOMON, 1949). Com isso, objetivou-se estudar a capacidade predatória da fase larval de C. caligata alimentada com B. Brassicae.

\section{MATERIAL E MÉTODOS}

Foram realizadas coletas de adultos de $C$. caligata no campus da Universidade Federal Rural da Amazônia UFRA, em Belém-PA. Em seguida, procedeu-se sua multiplicação no Laboratório de Bioecologia de Insetos (LABIN) do Instituto de Ciências Agrárias - ICA, pertencente à UFRA.

A capacidade predatória de larvas de $C$. caligata foi avaliada mediante fornecimento diário de B. brassicae, para os três instares, constituindo, dessa forma as densidades de presas (Tabela 1).

Tabela 1. Densidades de $B$. brassicae utilizadas nos experimentos de predação e resposta funcional da fase larval de C. caligata.

\begin{tabular}{cccc}
\hline \multirow{2}{*}{ DENSIDADES } & \multicolumn{3}{c}{ INSTARES } \\
\cline { 2 - 4 } & $1^{\mathbf{0}}$ & $2^{\text {o }}$ & $3^{\mathbf{o}}$ \\
\hline 1 & 4 & 14 & 36 \\
2 & 6 & 20 & 72 \\
3 & 8 & 26 & 114 \\
4 & 10 & 32 & 140 \\
5 & 12 & 38 & 180 \\
\hline
\end{tabular}

A quantidade de pulgão utilizada foi determinada através de um ensaio preliminar efetuado a $25 \pm 2{ }^{\circ} \mathrm{C}$, com 10 repetições, visando o fornecimento de um número de pulgões a capacidade de consumo diário em cada instar.

Os pulgões foram criados em folhas de couve Brassicae oleracea v. acephala. Aos 30 dias de germinadas, foram transplantadas para vasos plásticos com $22 \mathrm{~cm}$ de altura e 20 $\mathrm{cm}$ de diâmetro e colocadas em casa de vegetação, tendo como substrato terra preta e adubada com cama de aviário, permanecendo por mais 30 dias a fim de atingir suporte necessário para futura infestação.

Por ser uma cultura propícia ao ataque do pulgão, e devido a temperatura local sofrer pouca variação, viabilizou a reprodução desse inseto facilitando o fornecimento diário de pulgões-da-couve, que foram utilizados como alimento para larvas de $C$. caligata.

As observações foram realizadas diariamente, avaliando se os seguintes parâmetros: duração e viabilidade de cada instar, da fase larval e do período do ciclo completo de $C$. caligata. Os pulgões predados foram contificados diariamente juntamente com os sobreviventes, com auxílio de microscópio. Após a troca dos recipientes, forneceu se novamente as larvas o número conhecido de presas, conforme a densidade estabelecida.

O delineamento experimental utilizado foi inteiramente casualizado, com 5 tratamentos (densidades), e cinco repetições com duas larvas de $C$. caligata por repetição, totalizando 50 larvas.

Os resultados do experimento foram submetidos à análise de variância, comparadas pelo teste de Scott e Knott a 5\% de significância, quando pertinente, e estudos de regressão, através do ajuste das equações de acordo com os tratamentos utilizados. 


\section{RESULTADOS E DISCUSSÃO}

Em relação à capacidade predatória de larvas de $\mathrm{C}$. caligata, observou-se que, no primeiro instar, o consumo foi maior no intervalo entre 24 e 48 horas, em relação às primeiras 24 horas após a eclosão, passando de 2,0 para 6,0 pulgões, o que representa um aumento de cerca de $200 \%$. Nos instares subseqüentes ocorreu o inverso, verificando-se um maior consumo nas primeiras 24 horas após a ecdise, resultado também observado para toda fase larval. O consumo total de $B$. brassicae por larvas de segundo instar durante as 24 horas, após a ecdise, foi de 18 , para o terceiro, de 85 , reduzindo para cerca de 10 e 63 pulgões, respectivamente, no intervalo entre 24 e 48 horas.

A duração do primeiro, segundo, terceiro instares, fase larval, de pupa e larva/adulto de $C$. externa nas densidades médias (8,26 e114 pulgões), foi $6,0 \pm 0,03 ; 6,5 \pm 0,02 ; 14,0 \pm$ 0,$04 ; 26,5 \pm 0,07 ; 17,5 \pm 0,04 \mathrm{e}$ de $44,0 \pm 0,1$ dias, respectivamente.

Os resultados foram superiores em todos os estádios quando comparados aos apresentados por (BARBOSA et al., 2002) para $C$. everes. As variações encontradas entre os resultados obtidos e os registrados na literatura, podem ser atribuídas ao tipo de presa oferecida, influência exercida pelas condições de temperatura, umidade relativa, disponibilidade e qualidade do alimento, na duração das fases.

Observando o consumo médio diário, para os três instares e fase larval, constatou-se um aumento de maneira concomitante ao desenvolvimento da larva, com 3,9 $(7,6 \%)$ no primeiro instar, 9,2 (17,9\%) no segundo instar e 38,8 pulgões/dia (75\%) no terceiro instar, totalizando 51,4 pulgões consumidos diariamente durante toda a fase larval (tabela 2).

Tabela 2. Duração, consumo por dia e consumo total de Ceraeochrysa caligata alimentada com Brevicoryne brassicae, durante fases de desenvolvimento.

\begin{tabular}{cccc}
\hline Fases de Desenvolvimento & Duração (dias) & Pulgões consumidos/dia & Total de pulgões consumidos \\
\hline $1^{\circ}$ Instar & 6 & 3,9 & 23,5 \\
$2^{\circ}$ Instar & 6,5 & 9,2 & 59,8 \\
$3^{\circ}$ Instar & 14 & 38,8 & 543,5 \\
Fase Larval & 26,5 & 51,9 & 626,8 \\
Pupa & 17,5 & - & - \\
Larva/Adulto & 44 & - & - \\
\hline
\end{tabular}

Fonseca et al. (2000) verificaram para os três instares e fase larval de $C$. externa, alimentada com $S$. graminum, a 24 ${ }^{\circ} \mathrm{C}$, um consumo médio diário de 3,$1 ; 10,5 ; 76,7$ e de 29 pulgões, respectivamente, evidenciando um aumento superior a vinte vezes entre o consumo no primeiro e terceiro instares. Nesta pesquisa, observou-se, para esse aumento no consumo, cerca de dez vezes, quando comparados o primeiro e o terceiro instares, incrementando de 3,9 para 38,8 .

Para o consumo total, verificou-se uma relação direta entre o número de pulgões predados e o desenvolvimento larval, constatando-se um argumento no número de presas consumidas, atingindo o máximo no terceiro instar. $\mathrm{O}$ consumo no terceiro instar foi cerca de $87 \%$ do total verificado para a fase larval correspondendo a 543,5 e 626,8 presas, respectivamente, fato observado por Ribeiro (1988), Chakrabarti et al. (1991), López (1996), Carvalho et al. (1997), Fonseca et al. (2000, 2001) e Tiraboschi et al (2001). O número total de presas consumidas no primeiro, segundo, terceiro instares e fase larval de $C$. externa foram de 23,5 ; 58,$8 ; 543,5$ e 626,8 pulgões, correspondendo a 3,$8 ; 9,5 ; 86,7$ e $100 \%$, respectivamente.
Os resultados referentes à predação de larvas de primeiro e segundo instares, quando comparados ao obtidos por Ribeiro (1988) com 52,8 e 81,8, respectivamente, para larvas de C. externa alimentadas com A. gossypii, a, $25^{\circ} \mathrm{C}$, mas semelhantes aos verificados por Fonseca et al. (2000), quando as larvas foram alimentadas com $\mathrm{S}$. graminum, correspondendo a 13,7 e 34,7 presas, respectivamente, a 24 ${ }^{\circ} \mathrm{C}$. Da mesma forma para os dados de Maia (2003) para $C$. externa.

Santa-cecília et al. (2001) constataram que apesar da alta viabilidade obtida para os estágios imaturos de C. externa alimentada com cochonilha-do-abacaxi, dysmicoccus brevipes (Cockerell, 1893) (Hemiptera: Pseudococcidae), a duração das fases foi prolongada em relação a de larvas alimentadas com ovos de A. kuehniella.

Verificou-se, para as larvas dos três instares e fase larval, um maior consumo em função do aumento na densidade. $\mathrm{Na}$ maior densidade obteve-se um resultado significativamente superior quanto ao número de afídeos predados, diferindo das duas densidades abaixo e, estas, das duas inferiores, as quais não variaram significativamente entre si (Figura 1).

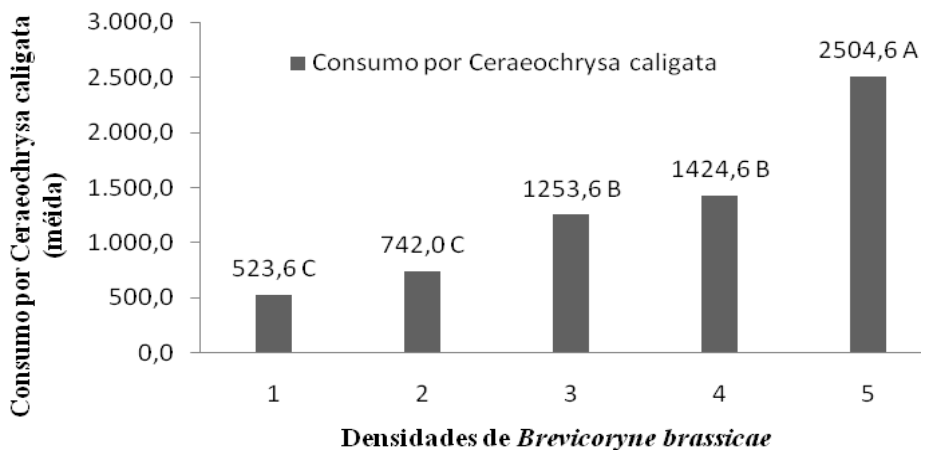

Figura 1 - Número total de Brevicoryne brassicae consumidos por larvas de Ceraeochrysa caligata, em função da densidade de pulgões. Temperatura de $25+0,2^{\circ} \mathrm{C}$, UR $85+10 \%$, fotofase de 12 horas. 
Zheng et al. (1993) verificaram, para larvas de Chrysoperla cárnea (Stephens, 1836) alimentadas com ovos do piralídeo Anagasta kuehniella (Zeller, 1879), um consumo cerca de duas vezes maior nas densidades superiores à capacidade predatória, quando comparado às inferiores, aproximando-se aos resultados obtidos para maior densidade.

No que se refere ao efeito da densidade de B. brassicae sobre a velocidade de desenvolvimento de $C$. caligata, verificou-se, de uma maneira geral, que a duração obtida para a fase larval, aumentou em função do aumento na densidade.

$\mathrm{Na}$ menor densidade, a duração do primeiro instar foi significativamente menos, evidenciando o efeito da densidade de $B$. brassicae sobre a duração dessa fase. Para segundo instar, as duas densidades maiores foram significativamente diferentes das demais, causando aumento de $50 \%$ na duração desses instares entre a menor e a maior densidade. No caso do terceiro, a velocidade de desenvolvimento nas duas densidades menores não variou significativamente, assim como entre as densidades 3 e 4 , porém, aumentou significativamente ao aumento na densidade, sendo de 35,6\% a diferença entre as densidades 1 e 2 , e a densidade 5. Não houve efeito significativo da densidade de pulgões sobre a duração da fase de pupa, em relação ao período larva/adulto, a menor duração foi observada na menor densidade. A duração de o terceiro instar foi significativamente maior na densidade máxima estudada, ouse já, apesar da saciação mais rápida do predador (GARCIA 1990), e das necessidades energéticas sendo supridas mais rapidamente o que, possivelmente explica a maior velocidade de desenvolvimento, as larvas passaram maior tempo se alimentando, o que, do ponto de vista de controle biológico, é significativamente importante (Tabela 3).

Tabela 3. Duração em dias ( \pm EP) dos três instares, fase larval, fase de pupa e de larva-adulto de $C$. caligata, em função da densidade de $B$. brassicae Temperatura de $25 \pm 2{ }^{\circ} \mathrm{C}$, UR $85 \pm 10 \%$ e fotofase de horas.

\begin{tabular}{ccccccc}
\hline \multirow{2}{*}{$\begin{array}{c}\text { Densidade } \\
\text { de presas }\end{array}$} & \multicolumn{3}{c}{ Duração dos instares (dias) } & Fase larval & Pupa & Larva adulto \\
\cline { 2 - 7 } & \multicolumn{1}{c}{$1^{\mathbf{0}}$} & $2^{\mathbf{0}}$ & $3^{\text {o }}$ & & & \\
\hline 1 & $3,5 \pm 0,2 \mathrm{~A}$ & $4,5 \pm 0,2 \mathrm{~A}$ & $11,8 \pm 0,4 \mathrm{~A}$ & $19,8 \pm 0,2 \mathrm{~A}$ & $16,0 \pm 0,2 \mathrm{~A}$ & $35,8 \pm 0,4 \mathrm{C}$ \\
2 & $5,0 \pm 0,2 \mathrm{~B}$ & $5,0 \pm 0,2 \mathrm{~A}$ & $11,8 \pm 0,2 \mathrm{~A}$ & $21,8 \pm 0,1 \mathrm{~A}$ & $17,5 \pm 0,3 \mathrm{~A}$ & $39,3 \pm 0,3 \mathrm{C}$ \\
3 & $6,0 \pm 0,1 \mathrm{~B}$ & $6,5 \pm 0,2 \mathrm{AB}$ & $14,0 \pm 0,3 \mathrm{~B}$ & $26,5 \pm 0,2 \mathrm{~B}$ & $17,5 \pm 0,3 \mathrm{~A}$ & $44,0 \pm 0,2 \mathrm{~B}$ \\
4 & $7,0 \pm 0,2 \mathrm{BC}$ & $8,5 \pm 0,1 \mathrm{C}$ & $14,0 \pm 0,3 \mathrm{~B}$ & $29,5 \pm 0,5 \mathrm{BC}$ & $17,3 \pm 0,4 \mathrm{~A}$ & $46,8 \pm 0,5 \mathrm{~B}$ \\
5 & $7,3 \pm 0,2 \mathrm{C}$ & $9,0 \pm 0,3 \mathrm{C}$ & $16,0 \pm 0,3 \mathrm{C}$ & $32,2 \pm 0,5 \mathrm{C}$ & $17,0 \pm 0,5 \mathrm{~A}$ & $49,3 \pm 0,5 \mathrm{~A}$ \\
\hline $\mathrm{F}$ & 3,77 & 0,85 & 3,62 & 9,78 & 0,88 & 6,98 \\
\hline $\mathrm{CV}(\%)$ & 11,7 & 14,62 & 17,74 & 7,13 & 9,29 & 3,65 \\
\hline
\end{tabular}

Maia (2003), obteve resposta tipo II para larvas de C. externa alimentadas com $R$. maidis, porém, não foi caracteristicamente marcante. A resposta tipo II, determinada para as larvas de C. externa por Maia (2003), foi a mesma verificada por Fonseca et al. (2000) para esse mesmo crisopídeo alimentado com $S$. graminum, evidenciando a adaptação desse predador a diferentes espécies de presas.

\section{CONCLUSÃO}

Houve influencia da densidade da presa sobre a sobrevivência e a duração das fases de desenvolvimento de Ceraeochrysa caligata;

$\mathrm{O}$ aumento no consumo de Brevicoryne brassicae por larvas de $C$. caligata foi de maneira concomitante ao aumento de tamanho das larvas do predador e da densidade;

O afídeo $B$. brassicae é uma presa viável para a criação massal de $C$. caligata, nas condições estudadas, sendo adequada pela facilidade de sua criação em larga escala.

\section{REFERÊNCIAS BIBLIOGRÁFICAS}

BARBOSA, L. R.; FREITAS, S.; AUAD, A. M. Capacidade reprodutiva e viabilidade de ovos de Ceraoechrysa everes (Banks, 1920) (Neuroptera: Chrysopidae) em diferentes condições de acasalamento. Ciência e Agrotecnologia, Lavras, v. 26, n. 3, p. 466-471, 2002.

CATI (2009). Levantamento sensitório das unidades de produção agropecuárias do Estado de São Paulo - Lupa. Dados consolidados do Estado 2007/2008. Disponível em:

<http://www.cati.sp.gov.br/projetolupa/dadosestado/Dad osEstaduais.pdf>. (acesso 29 jun. 2009).

CÉSAR P.S.; ÉDSON P.T. Resistência de variedades de couve a Brevicoryne brassicae (L.) (Hemiptera: Aphididae) Bioikos, Campinas, 24(2):113-118, jul./dez., 2010.

CIVIDANES, F.J. Uso de graus-dia em entomologia: com particular referência ao controle de percevejos pragas de soja. Jaboticabal, FUNEP, 31p. 2000.

FIGUEIRA, L.K. et al. Biologia e exigências térmicas de Chrysoperla externa (Hagen, 1861) (Neuroptera: Chrysopidae) alimentada com ovos de Alabama argillacea (Hübner, 1818) (Lepidoptera: Noctuidae). Cien. Agrotecn., Lavras, v. 24, n. 2, p. 319-326, 2000.

FONSECA, A.R.; CARVALHO C.F.; SOUZA, B. Resposta funcional de Chrysoperla externa (Hagen, 1861) (Neuroptera: Chrysopidae) alimentada com Schizaphis graminum (Rondani, 1852) (Homoptera: Aphididae). An. Soc. Entomol. Brasil 29: 309-317. 2000.

FONSECA, A.R.; CARVALHO, C.F.; SOUZA, B. Capacidade predatória e aspectos biológicos das fases imaturas de Chrysoperla externa (Hagen, 1861) (Neuroptera: Chrysopidae) alimentada com Schizaphis graminum (Rondani, 1852) (Hemiptera: Aphididae) em diferentes temperaturas. Ciênc. Agrotec. 25: 251-263. 2001. 
HADDAD, M. L.; PARRA, J. R. P. Métodos para estimar os limites térmicos e a faixa ótima de desenvolvimento das diferentes fases do ciclo evolutivo de insetos. Piracicaba, Fundação de Estudos agrários Luiz de Queiroz. 12p. (Boletim da série Agricultura e desenvolvimento), 1984.

HADDAD, M.L.; PARRA, J.R.P.; MORAES, R.C.B. Métodos para estimar os limites térmicos inferior e superior de desenvolvimento dos insetos. Piracicaba, FEALQ, 29p. 1999.

LAVAGNINI, T.C.; FREITAS, S. Capacidade reprodutiva e longevidade de Chrysoperla externa cujas fases imaturas foram submetidas à ambientes com diferentes temperaturas de criação. Nucleus, v.9, n.1, abr. 2012.

LIMA, J. O. G.; GRAVINA, G. A. Failure of imidacloprid and thiacloprid to control the guava-psyllid, Triozoida limbata (Enderlein) (Hemiptera: Psyllidae). Ciência e Agrotecnologia, v. 33, Edição Especial, p. 1888-1891, 2009.

MAIA, W.J.M.S. et al. Exigências térmicas de Chrysoperla externa (Hagen, 1861) (Neuroptera: Chrysopidae) alimentada com Schizaphis graminum (Rondani, 1852) (Homoptera: Aphididae) em condições de laboratório. Cien. Agrotecn., Lavras, v. 24, n. 1, p .81-86, 2000.

MAIA, W.J.M.S.et al. Capacidade predatória e aspectos biológicos de Chrysoperla externa (Hagen, 1861) (Neuroptera: Chrysopidae) alimentada com Rhopalosiphum maidis (Fitch, 1856) (Hemiptera: Aphididae). Ciência e Agrotecnologia, v.28, p.12591268, 2004

MOURA, A. P. et al. Toxicidade de pesticidas recomendados na produção integrada de maça (PIM) a populações de Chrysoperla externa (Hagen) (Neuroptera: Chrysopidae). Neotropical Entomology, v. 38, n. 3, p. 395-404, 2009.

MOURA, A. P.; MOURA, D. C. M. Levantamento e flutuação populacional de parasitoides de moscasdasfrutas (Diptera: Tephritidae) de ocorrência em goiabeira (Psidium guajava L.) em Fortaleza, Ceará. Arquivos do Instituto Biológico, v. 78, n. 2, p. 225-231, 2011.

OLIVEIRA, J.E. de M.; TORRES, J.B.; CARRANOMOREIRA, A.F.; RAMALHO, F.S. Biologia de Podisus nigrispinus predando lagartas de Alabama argillacea em campo. Pesquisa Agropacuária Brasileira, v.37, n.1, p.7-14, 2002.

PAZINI, W. C.; GALLI, J. C. Redução de aplicações de inseticidas através da adoção de táticas de manejo integrado do Triozoida limbata (Enderlein, 1918) (Hemiptera: Triozidae) em goiabeira. Revista Brasileira de Fruticultura, v. 33, n. 1, p. 66-72, 2011.

PESSOA, L. G. A.; LEITE, M. V. de; FREITAS, S.; GARBIN, G. C. Efeito da variação da temperatura sobre o desenvolvimento embrionário e pós-embrionário de Ceraeochrysa paraguaria (Navás) (Neuroptera:
Chrysopidae). Arquivos do Instituto Biológico, São Paulo, v. 71, n. 4, p. 473-476, out./dez. 2004.

SILVA, G.A.; CARVALHO, C.F.; SOUZA, B. Aspecto biológicos de Chrysoperla externa (Hagen, 1861) (Neuroptera: Chrysopidae) alimentada com lagartas de Alabama argillacea (Hübner, 1818) (Lepidoptera: Noctuidae). Ciência Agrotec., Lavras, v.26, n.4, p.682698, jun/ago., 2002.

SILVEIRA NETO, S. Armadilha luminosa. Piracicaba: USP, 1986. 8p. (USP-ESALQ. Informativo Tecnico, 3). Embrapa Meio Norte / UEP-Parnaíba.

SOUZA-FILHO, Z. A. et al. Endemic parasitoids associated with Anastrepha spp. (Diptera: Tephritidae) infesting guava (Psidium guajava) in Southern Bahia, Brazil. Florida Entomologist, v. 90, n. 4, p. 783- 785, 2007.

STEIN, C.P.; TEIXEIRA, E.P. Resistência de variedades de couve a Brevicoryne brassicae (L.) (Hemiptera: Aphididae). Bioikos, Capinas, n. 24(2), p. 113-118, jul./dez., 2010.

VAIL, P.V. et al. History of biological control programs in the United States Department of Agriculture. American Entomologist, Lanham, v. 47, p. 24-50, 2001. 